\title{
Fuzzy Clustering for Military Decision Analysis with Remote Sensing Information
}

\author{
Jifeng Yang \\ Navy Submarine Acadamy
}

\author{
Yongzhi Liu \\ Navy Submarine Acadamy
}

\author{
Bingjie Liu \\ Navy Submarine Acadamy
}

\author{
Weiliang Chen \\ Navy Submarine Acadamy
}

\begin{abstract}
In ocean military actions, there are many environmental factors to influent the military decision. In this paper, the fuzzy C-means(FCM) clustering is used to evaluate the influents of ocean environment, including sea winds, sea wave, sea current and the sea surface temperature gradient in military game. To different military application, the influence coefficient are different, in this paper a weighting exponent is import to estimate the influence grade of the entire ocean environment. The experimental results demonstrate that this algorithm's utility in the classification of ocean area of military game.
\end{abstract}

Keywords-Fuzzy C-means Clustering; Sea Winds; Wave; Current; Sea surface temperature gradient

\section{INTRODUCTION}

In ocean military decisions,the ocean environment including the atmosphere and hydrographic environment is especially important compared to the other military actions. For example, the sea wind and sea wave have a large influence in the operation of ship, the temperature gradient always decides the location of ocean fronts and meso-scale eddies, which can have a large influence in the operating and precision of sonar. The sea current and the meso-scale ocean features may influence the blow efficiency of undersea weapon, such as torpedo and underwater mine. The sea current, sea wave, sea wind and the meso-scale ocean features may also influence the lunch of the undersea guided missile.

To find a method to analysis all the ocean environment factors and decides the best military operational sea area is an important subject in military decision.

In this paper, the ocean environment information is obtained by remote sensing ways, including space-borne, airborne, and fused remote sensing data. Then the fuzzy Cmeans clustering method is used to analysis all the information, clustering the factors and finally divides the authorized sea area into several areas, which leveled the fitness of the military action.

\section{THE FUZZY C-MEANS CLUSTERING}

To the data set formed by $\mathrm{N}$ p-dimensional feature vectors, that is, $X=\left\{x_{1}, x_{2}, \ldots, x_{N}\right\}, x_{k} \in R^{p}$, $k=1,2, \ldots, N$.

The fuzzy c-partition of the given data set is a crisp partition matrix $U=\left[u_{i j}\right], \quad i=1,2, \ldots, C$ and $j=1,2, \ldots, N$.The aim of the Fuzzy C-means algorithm is to find an optimal fuzzy c-partition by evolving the fuzzy partition $U=\left|u_{i j}\right|$ iteratively and computing the cluster centers. The clustering algorithm is based on a least-squared criterion $^{[1]}$ :

$$
J_{m}(U, V)=\sum_{i=1}^{C} \sum_{j=1}^{N} u_{i j}{ }^{m}\left\|x_{j}-v_{i}\right\|^{2}
$$

Where $U$ is a fuzzy c-partition of data set $\mathrm{X}$, $u_{i j} \in[0,1]$ is the membership of feature vector $x_{j}$ to cluster $C_{i}$. It means that each vector $x_{j}$ belongs exactly to one of the $\mathrm{C}$ clusters. It satisfies the following constraint given by Equation (2) and (3).

$$
\begin{gathered}
\sum_{i=1}^{C} u_{i j}=I \quad \text { For } j=1,2, \ldots, N \\
0<\sum_{i=1}^{C} u_{i j}<N \quad \text { For } i=1,2, \ldots, C
\end{gathered}
$$

Where $m \in[1, \infty)$ is the weighting exponent on each fuzzy membership, the larger $m$ is, the fuzzier the partition. When $m=1$, the fuzzy C-means Clustering is the same as the Hard C-means Clustering ${ }^{[2][3][4][5]}$.

The FCM algorithm tries to minimize $J_{m}$ by iteratively updating the partition matrix using the following equations:

$$
\begin{array}{r}
v_{j k}=\frac{\sum_{i=1}^{n}\left(u_{i k}\right)^{m} X_{j}}{\sum_{i=1}^{n}\left(u_{i j}\right)^{m}} \\
u_{i j}=\left[\sum_{i=1}^{c}\left(\frac{d_{i j}}{d_{j k}}\right)^{2 /(m-1)}\right]^{-1}
\end{array}
$$

As the GLCM statistic elements take different weightiness in instrument corrosion, we must treat them differently in clustering. So we can define an weightiness vector $w$. In this paper, there are four elements, so the weightiness vector has four elements too. And the vector satisfies the constraint $0<w_{n}<1$, and $\sum_{n=1}^{4} w_{n}=1$. The distance can then calculate by the following formula:

$$
d_{i j}=\left[w_{j}\left(r_{i j}-v_{j k}\right)\right]^{2}
$$


Where $i=1,2, \ldots, C$ and $j=1,2, \ldots, N$.

\section{REMOTE SENSING OCEAN INFORMATION CLUSTERING ALGORITHM}

In this study, there are four different ocean elements namely the sea current, the sea wave, the sea wind and the sea surface temperature gradient. All the ocean elements are collected by remote sensing and turned into grid images. The aim of the clustering is divide the ocean area into several classes, each of which has some similar influence to the instrument corrosion on the ship and the weapon operating on it.

The clustering algorithm is combined with the following steps:

Step1: Determining the number of cluster $C$ and $\mathrm{m}$ value, some researches ${ }^{[6][7]}$ indicate that the best value of $m$ is in $[1.5,2.5]$, and $m=2$ is a reasonable value. And determine the given converging error, $\varepsilon>0$ (such as $\mathcal{E}=0.001)^{[8]}$.

Step2: Make all the ocean elements into one dimension along the longitude, and get them together into an $X$ array by rows. As there are four ocean elements, the row number of $X$ array is four.

Step3: Make up an original fuzzy array $\left(u_{i j}{ }^{0}\right)_{c \times 4}$, where the elements in $u_{i j}{ }^{0}$ is not equal and satisfies the constraint given by Equation (10) and (11).

Step4: Put the fuzzy array $\left(u_{i j}{ }^{0}\right)_{c \times 4}$ into formula (10) to calculate the core array $v_{j k}{ }^{0}$.

Step5: Put the original fuzzy array $v_{j k}{ }^{0}$ into formula (11) to calculate the fuzzy array $\left(u_{i j}{ }^{1}\right)_{c \times 5}$.

Step6: Put the fuzzy array $\left(u_{i j}{ }^{1}\right)_{c \times 4}$ into formula (10) to calculate the core array $v_{j k}{ }^{1}$.

Step7: Calculate the distance of every element between $\left(u_{i j}\right)_{\propto 4}$ and $\left(u_{i j}{ }^{0}\right)_{c \times 4}$, if $\max \left\|u_{i j}{ }^{1}-u_{i j}{ }^{0}\right\|>\mathcal{E}$, return to step6.

Step8: Calculate the distance of every element between $v_{j k}{ }^{1}$ and $v_{j k}{ }^{0}$, if $\max \left\|v_{i j}{ }^{1}-v_{i j}{ }^{0}\right\|>\varepsilon$, return to step7.

Step9: Calculate the classification array $H$, with the following formula:

$$
H=\operatorname{round}\left([1: c]^{*} U\right)
$$

With the array $\mathrm{H}$, we can get the FCM clustering result and divide the original array $\mathrm{X}$ into a one row 4 number array, which corresponding to the 4 types of the military action area.

\section{MILITARY DECISION ANALYSIS}

In this paper, we use four kinds of remote sensing data, including sea current speed, the sea wave height, the sea wind speed and the sea surface temperature gradient. They are pretreated and statistic by times. Here use the assembly average of March from 2000 to 2010 and the sea area of them are the longitude is from $105^{\circ} \mathrm{E}$ to $145^{\circ} \mathrm{E}$ and the latitude is form $0^{\circ} \mathrm{N}$ to $40^{\circ} \mathrm{N}$. The assembly average sea wave height of March is shown in Figure.1. The assembly average Sea Wind is shown in figure.2. The assembly average Sea temperature gradient is shown in Figure.3. The assembly average sea current is shown in Figure.4.

In figure.2, there are some abnormal area belong the shore, because we can't use the remote sensing data to reconvert the wind speed correctly.

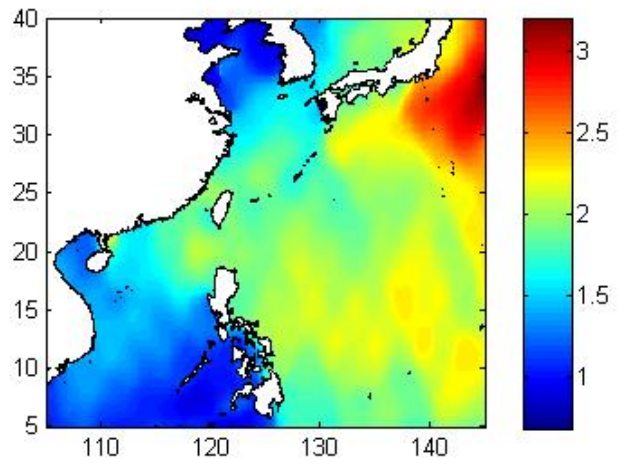

Figure 1. Sea wave height

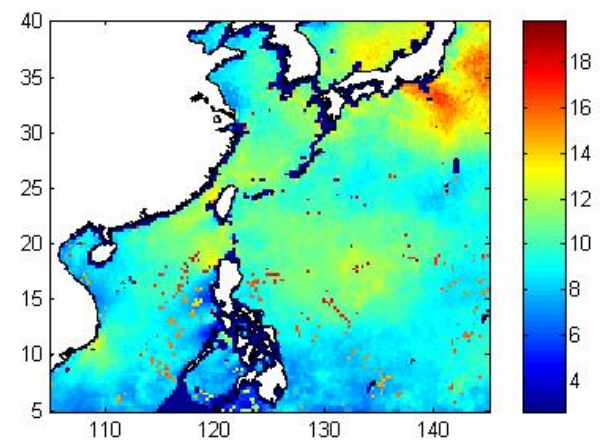

Figure 2. Sea wind speed 


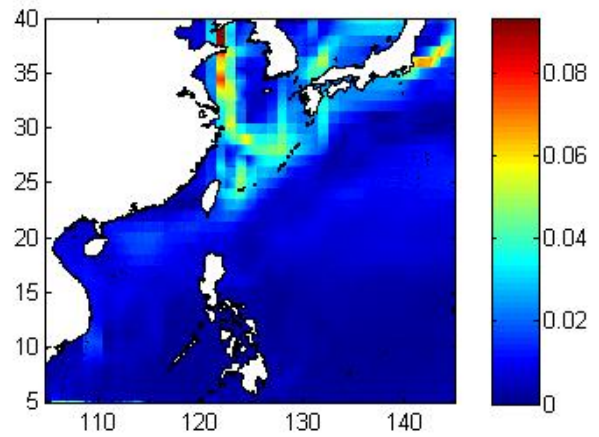

Figure 3. SST gradient

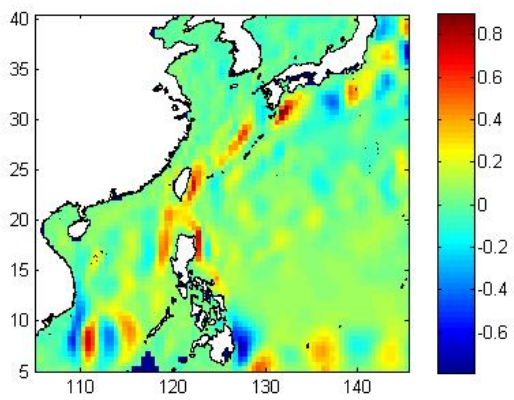

Figure 4. Sea current speed

To some military use, for example the operation of the ships, the sea wave and sea winds may the main influence, if the weight of the sea wave is 0.8 and the sea wind is 0.2 , the cluster number is 4 , then the cluster result of the algorithm stated in is paper is shown in Figure.5. The target area is divided into four type of areas, use four different color to mark them, the number one areas use dark blue to mark, the number two areas use blue, the number three use yellow and the number four use dark red. Because of the abnormal data in sea wind speed of shore, the fourth area of the clustering can be out considering in military decision. The smaller the number of the areas, the better the environment for the ship operating.

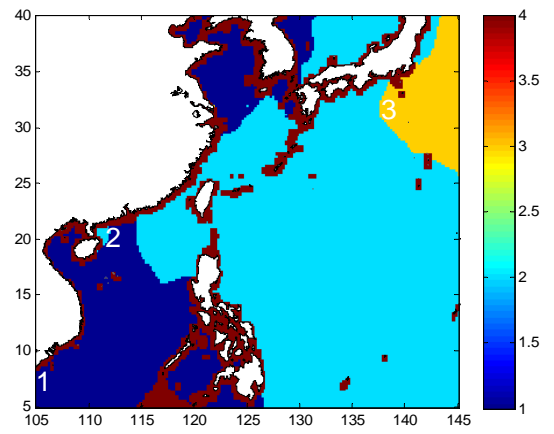

Figure 5. The cluster result for ship operating
To some other military use, more environmental factors should be considered. For example, the lunch of the undersea guided missile. All the four factors stated are included, if the weight of the sea wave is 0.5 ,the sea current is 0.1 , the sea wind is 0.2 and the sea surface temperature gradient is 0.1 , the cluster number is 4 , then the cluster result is shown in Figure.6. Because of the abnormal data in sea wind speed of shore, the fourth area of the clustering can be out considering in military decision. The smaller number of the areas, the better the environment for missile lunching.

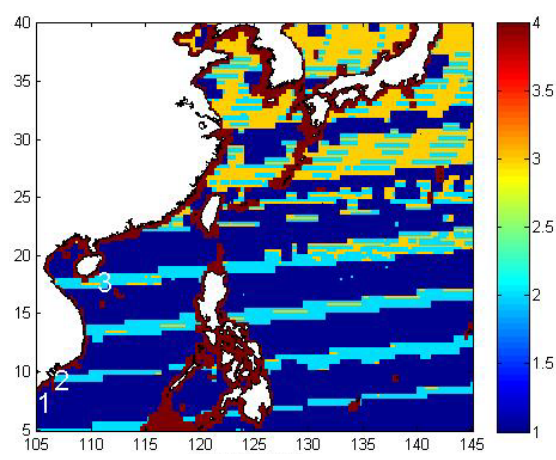

Figure 6. The cluster result for ship operating

\section{CONCLUSIONS}

In this paper, an automatic subsidiary military decision analysis system using remote sensing information based on fuzzy C-mean crusting is proposed. The weight of different environment in the clustering algorithm is just simply analyzed. In the following studies, we can use hydrodynamic model and other coherent theory to calculate the influence of the environments more accurate. The experimental results show that the algorithm stated in this paper is reasonable and has great potential in subsidiary decision analysis

\section{REFERENCES}

[1] Kachouie, Nezamoddin N., Alirezaie, Javad, Raahemifar, Kaamran. Genetically derived fuzzy C-means clustering algorithm for segmentation. Canadian Conference on Electrical and Computer Engineering, 2003, v 2, 1119-1122

[2] Ronhua Tao, Jie Chen, Biao chen, Chuihua Liu. GLCM and Fuzzy Clustering for Ocean Features Classification. Proc of the 2010 IEEE Int'l.Conf on MVHI,1340-1343

[3] Xizhao Wang, Yadong Wang, Lijuan Wang. Improving fuzzy cmeans clustering based on feature-weight learning. Pattern Recognition Letters 25(2004) 1123-1132

[4] Krinidis, S. A Robust Fuzzy Local Information C-Means Clustering Algorithm. Image Processing, IEEE Transactions on Date of Publication: May 2010, Volume: 19, Issue: 5 ,Page(s): 1328 - 1337

[5] Sivakumar, S.,Chandrasekar, C. Lungs image segmentation through weighted FCM. Recent Advances in Computing and Software Systems (RACSS), 2012 International Conference, Page(s): 109 113

[6] Zhang D, Kamel M, Elmasry M. Fuzzy clustering neural network(FCNN): competitive learning and parallel architecture. Intellingent and Fuzzy Systems, v 2, 289-298(1994)

[7] Yu J, Huan HK, Tian SF. An efficient optimality test for the fuzzy Cmeans algorithm. Proc of the 2002 IEEE Int'l.Conf on Fuzzy Systems. 789-795(2002) 
[8] Tian Junwei, Huang Yongxuan. Histogram Constraint Based Fast FCM Cluster Image Segmentation. Industrial Electronics, 2007. ISIE 2007. IEEE International Symposium on Topic(s): Components, Circuits, Devices \& Systems ; Computing \& Processing (Hardware/Software) ; Power, Energy, \& Industry Applications. Page(s): 1623 - 1627 